08,04

\title{
Влияние решеточного и спин-фононного вкладов на температурное поведение расщепления основного состояния $\mathrm{Gd}^{3+}$ в $\mathrm{SrMoO}_{4}$
}

\author{
(С) А.Д. Горлов
}

Уральский федеральный университет, Институт естественных наук и математики, Екатеринбург, Россия

E-mail: Anatoliy.Gorlov@urfu.ru

(Поступила в Редакцию 24 мая 2017 г.)

Исследовано температурное поведение спектров ЭПР примесного центра $\mathrm{Gd}^{3+}$ в монокристаллах $\mathrm{SrMoO}_{4}$ в диапазоне температур $T=99-375 \mathrm{~K}$. Проведен анализ температурных зависимостей параметров спинового гамильтониана $b_{2}^{0}(T)=b_{2}(F)+b_{2}(L)$ и $P_{2}^{0}(T)=P_{2}(F)+P_{2}(L)$ (для $\mathrm{Gd}^{157}$ ), описывающих спектр ЭПР и дающих вклад в расщепление $\Delta E$ основного состояния $\mathrm{Gd}^{3+}$. В рамках суперпозиционной модели Ньюмена оценены значения $b_{2}(L)$ и $P_{2}(L)$, зависящие от температурного расширения статической решетки, затем выделены спин-фононные вклады $b_{2}(F)$ и $P_{2}(F)$, определяемые колебаниями ионов решетки. Анализ $b_{2}^{0}(T)$ и $P_{2}^{0}(T)$ указывает на положительный вклад спин-фононного взаимодействия, а модель локальных колебаний примесного кластера с близкими частотами $\omega$ хорошо описывает температурное поведение $b_{2}(F)$ и $P_{2}(F)$.

Работа выполнена в рамках государственного задания Минобрнауки России для Уральского федерального университета (3.6115.2017/8.9) на оборудовании Центра коллективного пользования „Современные нанотехнологии“ УрФУ.

DOI: 10.21883/FTT.2018.02.45388.169

\section{1. Введение}

Соединения молибдатов были и остаются интересными материалами для изучения, обусловленного их применением в практических устройствах. Хорошие оптические свойства, химическая инертность обусловили их применение в виде различных оптоэлектронных элементов в различных устройствах [1-3], лазерных решеток [4]. С развитием технологий криогенного детектирования интерес к соединениям молибдатов возpoc, a $\mathrm{CaMoO}_{4}$ наиболее используемый материал для некоторых устройств. $\mathrm{SrMoO}_{4}$, как сцинтиллятор в рентгеновском диапазоне, также проявляет более высокую эффективность, чем многие другие молибдаты, поэтому он может использоваться и считается дополнительным материалом [5]. Физические параметры кристаллов определяют эффективность устройств и существенным образом зависят от фононного спектра, меняющегося с температурой $(T)$. Теоретические модели, объясняющие такие температурные изменения, связанные с фононным спектром, еще несовершенны, поэтому новые экспериментальные результаты, из которых можно выделить спин-фононный вклад (СФВ), могут помочь в их развитии.

Данная работа является продолжением наших ЭПРисследований [6,7], посвященных анализу изменения параметров начального расщепления $\Delta E$ основного состояния примесных центров (ПЦ) $\mathrm{Gd}^{3+}$ в кристаллах $\mathrm{CaWO}_{4}$ и $\mathrm{CaMoO}_{4}$ за счет температурного изменения координат лигандов (implicit effect) и действию решеточных колебаний, или СФВ (explicit effect) $[8,9]$.
Кроме наибольшего из параметров спинового гамильтониана $(\mathrm{C \Gamma}) b_{2}^{0}$, определяющего расщепление $\Delta E$ для $\mathrm{SrMoO}_{4}: \mathrm{Gd}^{3+}$, в рассмотрение мы включили еще и квадрупольное взаимодействие $($ КВ $) P_{2}^{0}$. Известно, что $\mathrm{KB}$, определяемое градиентом электрического кристаллического поля (КП) лигандов на ПЦ, пропорционально величине этого поля $A_{2}^{0}[10,11]$. Следовательно, выделив СФВ из величин $b_{2}^{0}(T)$ и $P_{2}^{0}(T)$, можно ожидать, что при правильном описании спин-фононного взаимодействия некоторые модельные параметры этих зависимостей должно быть одинаковы.

\section{2. Экспериментальные результаты}

Кристалл $\mathrm{SrMoO}_{4}: \mathrm{Gd}^{3+}$ выращен методом Чохральского с примесью $0.02 \%$ по весу $\mathrm{Gd}_{2} \mathrm{O}_{3}$ (с природным содержанием изотопов) в шихте. Спектры ЭПР были записаны на спектрометре Bruker EMX plus в 3-cm диапазоне при разных $T$ и ориентациях внешнего магнитного поля Н. Разрешенная сверхтонкая структура (СТC), обязанная нечетному изотопу $\mathrm{Gd}^{157}$ (электронный спин $S=7 / 2$, ядерный спин $I=3 / 2$ ), наблюдалась на всех сигналах ЭПР в ориентациях $\mathbf{H} \| \mathbf{S}_{4}-$ главной оси кристалла и на нескольких - при $\mathbf{H} \perp \mathbf{S}_{4}$.

Спектр ЭПР от четных и нечетного изотопов прекрасно описывается спин-гамильтонианом, соответствующим локальной симметрии ПЦ $D_{2 d}$, т.е. $\mathrm{Gd}^{3+}$ замещает $\mathrm{Sr}^{2+}$ [12]. Численная минимизация среднеквадратичного отклонения экспериментальных и расчетных положений сигналов показала, что величины параметров $b_{n}^{m}(T)(n=2,4,6$ и $m=0,4,6)$ для четных и нечетного 
Таблица 1. Экспериментальные параметры спинового гамильтониана примесного иона $\mathrm{Gd}^{157}$ в $\mathrm{SrMoO}_{4}$ и расчетный решеточный вклад статической решетки в $Z_{2}(L)$ (в $\left.\mathrm{MHz}\right)$ при разных $T$

\begin{tabular}{|c|c|c|c|c|c|c|}
\hline \multirow[t]{2}{*}{$T, \mathrm{~K}$} & \multicolumn{4}{|c|}{ Параметры СГ } & \multicolumn{2}{|c|}{$\begin{array}{c}\text { Расчетный вклад } \\
\text { статической решетки }\end{array}$} \\
\hline & $b_{2}^{0}$ & $b_{4}^{0}$ & $b_{4}^{4}$ & $P_{2}^{0}$ & $b_{2}(L)$ & $P_{2}(L)$ \\
\hline $\begin{array}{r}99 \\
111 \\
125 \\
150 \\
175 \\
200 \\
250 \\
273 \\
298 \\
375\end{array}$ & $\begin{array}{l}-2496.0(3) \\
-2492.7(3) \\
-2489.4(4) \\
-2482.0(3) \\
-2472.7(3) \\
-2463.3(4) \\
-2443.0(3) \\
-2434.4(4) \\
-2427.3(4) \\
-2394.5(9)\end{array}$ & $\begin{array}{l}-39.29(15) \\
-39.10(15) \\
-39.09(30) \\
-38.84(30) \\
-38.55(29) \\
-38.18(33) \\
-37.73(3) \\
-37.40(23) \\
-37.20(22) \\
-36.2(5)\end{array}$ & $\begin{array}{l}-249.3(20) \\
-248.0(31) \\
-247.3(23) \\
-247.7(10) \\
-246.5(11) \\
-243.5(12) \\
-238.7(20) \\
-237.1(18) \\
-235.6(20) \\
-230.0(12)\end{array}$ & $\begin{array}{l}-57.3(4) \\
-57.3(4) \\
-57.2(5) \\
-57.2(4) \\
-57.0(4) \\
-56.9(5) \\
-56.7(4) \\
-56.4(5) \\
-56.3(4) \\
-55.9(6)\end{array}$ & $\begin{array}{l}-2519.7 \\
-2523.1 \\
-2527.4 \\
-2536.1 \\
-2545.9 \\
-2556.8 \\
-2581.2 \\
-2593.5 \\
-2605.7 \\
-2653.5\end{array}$ & $\begin{array}{l}-58.3 \\
-58.5 \\
-58.7 \\
-59.2 \\
-59.7 \\
-60.3 \\
-61.7 \\
-62.4 \\
-63.2 \\
-65.7\end{array}$ \\
\hline
\end{tabular}

Примечание. $g_{x}=g_{y}=1.9915(4), g_{z}=1.9918(3), b_{6}^{0}=0.16(10), b_{6}^{4}=6(8), b_{6}^{6}=2(6), A_{x}=A_{y}=16.2(3), A_{z}=16.2(3)$. Средняя ошибка в $b_{2}(L)$ за счет неточности параметров решетки $\sim 0.8 \%,(\sim 20 \mathrm{MHz})$, а в $P_{2}(L) \sim 2.2 \%(\sim 1.3 \mathrm{MHz})$.

изотопов $\mathrm{Gd}^{3+}$ в $\mathrm{SrMoO}_{4}$ практически совпадают в пределах ошибок измерений, причем близки и среднеквадратичные ошибки. Использование СГ для реальной локальной симметрии ПЦ $\mathrm{S}_{4}$ не приводило к уменьшению ошибок в определении параметров. В табл. 1 приведены их значения для $\mathrm{Gd}^{157}$ в $\mathrm{SrMoO}_{4}$.

\section{3. Модельные расчеты}

Определим $Z_{n}^{m}(T)=b_{n}^{m}(T)$ (либо $\left.P_{n}^{0}(T)\right)$ как экспериментально определенные константы СГ, а СФВ в диагональные параметры $(m=0) Z_{n}(F)=b_{n}(F)$ (либо $\left.P_{n}(F)\right)$. Тогда $[6,7]$

$$
Z_{n}(F)=Z_{n}^{0}(T)-Z_{n}(L) .
$$

Для $n=2 Z_{2}(L)=b_{2}(L)$ (либо $\left.P_{2}(L)\right)$ являются вкладами статической решетки при данной $T$. Величины $Z_{2}(L)$ при разных $T$ рассчитаем с помощью суперпозионной модели Ньюмена [13-15] в виде, представленном в работе [16].

3.1. Расчет параметров решетки. Для определения $b_{2}(L)$ и $P_{2}(L)$ нам необходимо знать $a$ и $c$ - параметры решетки для $\mathrm{SrMoO}_{4}$ в широком диапазоне $T$. В литературе нет таких данных для низких температур, однако приведены экспериментально измеренные параметры $a$ и $c$ для $\mathrm{SrMoO}_{4}$ в диапазоне $T=299-931 \mathrm{~K}$ [17]. Мы предположили, что зависимости $a(T)$ и $c(T)$ имеют полиномный вид, как в работе [18] ((6) для $\left.\mathrm{CaWO}_{4}\right)$

$$
l(T)=l_{0}\left(1+l_{1} T^{2}+l_{2} T^{3}+l_{3} T^{4}\right),
$$

где $l=a$ либо $c$. Используя в качестве опорных точек в процедуре fitting данные работы [17] и (2), мы получили зависимости $a(T)$ и $c(T)$ с параметрами (в $\AA)$ :

$$
\begin{gathered}
a_{0}=5.3888(16), \quad a_{1}=2.0438(199) \cdot 10^{-8}, \\
a_{2}=-2.045(245) \cdot 10^{-11}, \quad a_{3}=8.6(28) \cdot 10^{-15}, \\
c_{0}=11.9861(48), \quad c_{1}=4.6014(836) \cdot 10^{-8}, \\
c_{2}=-4.565(110) \cdot 10^{-11}, \quad c_{3}=1.85(12) \cdot 10^{-14} .
\end{gathered}
$$

Приведенные ошибки (цифры в скобках) соответствуют утроенному среднеквадратичному отклонению $3 \sigma$.

Чтобы проверить достоверность применения (2), мы провели аналогичную процедуру с данными работы [19] для $\mathrm{CaMoO}_{4}$, взятыми в диапазоне $T \sim 300-1273 \mathrm{~K}$. Полученные нами $a(T)$ и $c(T)$ неплохо совпадают с экспериментальными результатами [20] в области $T=10-300 \mathrm{~K}$. Используя (2) с определенными выше $a_{i}$ и $c_{i}$, мы рассчитали параметры решетки $\mathrm{SrMoO}_{4}$ при нужных $T$, а затем и сферические координаты $R, \theta, \varphi$ ближайших к $\mathrm{Gd}^{3+} 8$ ионов кислорода $\left(\mathrm{O}^{2-}\right)$ в чистой решетке $\mathrm{SrMoO}_{4}$. Координаты $x, y, z$ этих $\mathrm{O}^{2-}$ взяты из данных работы [21].

3.2. Оценка параметров $Z_{2}(L)$. Процедура расчета параметров $Z_{2}(L)$ в суперпозионной модели [13-16] аналогична описанной в $[6,7]$. В выражении

$$
Z_{2}(L)=\Sigma_{i} k_{i}(\theta)\left[Z_{2 p}\left(R_{0} / R_{i}\right)^{3}+Z_{2 s}\left(R_{0} / R_{i}\right)^{10}\right]
$$

„intrinsic“ параметры $Z_{2 p} \quad$ (равные $b_{2 p}=$ $=-1.289 \cdot 10^{4} \mathrm{MHz}$ либо $\left.P_{2 p}=-545.6 \mathrm{MHz} \quad[16]\right)$ являются вкладами от точечного заряда $\mathrm{O}^{2-}$. Координационные факторы $k_{i}(\theta)=n\left(3 \cos ^{2} \theta_{i}-1\right) / 2$. Поскольку ближайшие к $\mathrm{Gd}^{3+}$ кислороды разбиваются на 2 четверки, эквивалентные по вкладам в $b_{2}^{0}$ и $P_{2}^{0}$, то $i=1,2$, а $n=4$. Расстояния $\mathrm{Gd}^{3+}-\mathrm{O}^{2-}$ рассчитано по формуле $R_{i}=R+\left(r-r_{h}\right) / 2$ [22], где $r, r_{h}$ - ионные радиусы примесного и замещаемого иона, взятые из таблиц 
Таблица 2. Модельные параметры температурных зависимостей $Z_{n}^{m}(T)$ и $Z_{2}(F)$ (в $\left.\mathrm{MHz}\right)$ для $\mathrm{Gd}^{3+}{ }_{\text {в } \mathrm{SrMoO}_{4}}$

\begin{tabular}{c|l|l|l}
\hline \multirow{2}{*}{ Зависимость } & \multicolumn{3}{|c}{ Параметры модели } \\
\cline { 2 - 4 } & \multicolumn{1}{|c}{$Z_{n}^{m}(0)$} & \multicolumn{1}{|c}{$Z_{n}(0)$} & $\omega \cdot 10^{-13}, \mathrm{rad} / \mathrm{s}$ \\
\hline$b_{2}^{0}(T)$ & $-2506.1(20)$ & $50.0(18)$ & $3.15(10)$ \\
$P_{2}^{0}(T)$ & $-57.4(46)$ & $1.65(48)$ & $5.58(87)$ \\
$b_{4}^{0}(T)$ & $-39.6(8)$ & $1.3(1)$ & $2.73(13)$ \\
$b_{4}^{4}(T)$ & $-250(5)$ & $16.3(51)$ & $4.6(8)$ \\
$b_{2}(F)$ & - & $126.8(96)$ & $3.4(2)$ \\
$P_{2}(F)$ & - & $4.9(5)$ & $3.47(50)$
\end{tabular}

Примечание. Ошибки в параметрах соответствуют $3 \sigma$.

Шеннона [23]. Параметры $Z_{2 s} \quad\left(b_{2 s}=1.008 \cdot 10^{4} \mathrm{MHz}\right.$, a $\left.P_{2 s}=555.8 \mathrm{MHz}\right)$, т. е. вклады от перекрывания и ковалентности на расстоянии $R_{0}=2.34 \AA$ [16], определены для $\mathrm{Gd}^{3+}$ в $\mathrm{SrMoO}_{4}$ следующим образом. Из зависимостей экспериментальных величин $b_{2}^{0}(T)$ и $P_{2}^{0}(T)$ в модели „жесткой решетки“ согласно (2)-(4) из [24] получены величины $Z_{2}^{0}(R L)$. Как показано в $[6,7]$ значения $Z_{2}^{0}(R L)=b_{2}^{0}(0)$ либо $P_{2}^{0}(0)$ (далее $\left.Z_{2}^{0}(R L)=Z_{2}^{0}(0)\right)$, т.е. близки к величинам экспериментальных параметров при $T \sim 1.8-4.2 \mathrm{~K}$, поскольку фононный вклад в этой области $T$ очень мал [6-9,25-27]. Заметим также, что эти величины получаются практически равными при использовании любой из моделей параметризации, изложенных в работах [24-27]. Определив согласно (2) $a$ и $c$ при $T \sim 2 \mathrm{~K}$, затем $R_{i}, \theta_{i}$ и $\varphi_{i}$ для кластера $\mathrm{Gd}^{3+}-8 \mathrm{O}^{2-}$ и используя (3), можно легко получить $b_{2 s}$ и $P_{2 s}$. В табл. 1 приведены расчетные значения $b_{2}(L)$ и $P_{2}(L)$ при нужных температурах, а в табл. 2 даны параметры $b_{2}^{0}(0)$ и $P_{2}^{0}(0)$.

\section{4. Температурная зависимость $Z_{2}(F)$ и обсуждение результатов}

Выделим из наших экспериментальных данных зависимость от температуры $b_{2}(F)$ и $P_{2}(F)$, который определим согласно (1) и данных табл. 1. Видно, что $b_{2}(F)$ и $P_{2}(F)$ положительны. Зависящее от температуры СФВ, влияющее на начальное расщепление $\Delta E$ уровней энергии для ПЦ в кристаллах, можно попытаться описать тремя наиболее известными теоретическими моделями [25-27]. В этих моделях предполагается доминирующий влияние оптических фононов [25], либо акустических [26] в СФВ. В модели [27] показано, что основную роль в фононно-индуцированном вкладе (или в СФВ) играют локальные оптические колебания примесного кластера. Хотя каждой модели соответствует своя функциональная температурная зависимость для $Z_{2}(F)$, в области $T>200 \mathrm{~K}$ все они дают практически линейную зависимость от $T$.

4.1. Температурная зависимость $b_{2}(F)$. Анализ показал, что в моделях $[25,26]$ зависимость фо- нонно-индуцированного вклада $b_{2}(F)$ от $T$ можно описать только тремя подгоночными параметрами, один из которых не имеет теоретического обоснования. С другой стороны, модель локальных колебаний [27] с двумя параметрами очень хорошо описывает зависимость $b_{2}(F)$ от $T$ выражением

$$
Z_{2}(F)=Z_{2}(0)[\operatorname{coth}(\omega / 2 k T)-1]
$$

где $Z_{2}(0)=b_{2}(0)-$ вклад за счет „нулевых колебаний“ решетки, $\omega$ - частота локальных колебаний примесного кластера, $k$ - постоянная Больцмана. На рисунке показана зависимость $b_{2}(F)$ от $T$ (кривая 1 ), полученная в процедуре genfit. Точки на кривой определены согласно (1), а в табл. 2 приведены модельные параметры, причем в скобках приведены их отклонения при $3 \sigma$.

4.2. Температурная зависимость $P_{2}(F)$. Хотя изменения $P_{2}^{0}(T)$ малы тем не менее рассчитав $P_{2}(L)$, мы видим, что фононно-индуцированный вклад $P_{2}(F) \sim 7 \mathrm{MHz}$ при $T \sim 300 \mathrm{~K}$. Как и для $b_{2}(F)$ в выражениях для температурной зависимости $P_{2}(F)$ в моделях $[25,26]$ требуются три подгоночных параметра в процедуре genfit, а модель локальных колебаний [27] хорошо описывает $P_{2}(F)$ согласно (4) (на рисунке это кривая 2). Модельные параметры даны в табл. 2, приведенные ошибки определены также при $3 \sigma$.

Отношение изменений решеточного вклада $\Delta b_{2}(L)=$ $=\left|b_{2}(L)-b_{2}^{0}(0)\right|$ к $\left|b_{2}(F)\right| \sim 0.56$ при $T=298 \mathrm{~K}$, причем $\Delta b_{2}(L)<0$. Это значит, что вклад в температурное изменение $b_{2}^{0}(T)$ локальных колебаний узлов примесного кластера в 1.7 раза больше, чем изменение $\Delta b_{2}(L)$ из-за расширения статической решетки. Аналогичные оценки для КВ дают $\Delta P_{2}(L)=P_{2}(L)-P_{2}^{0}(0)<0$, а $\left|\Delta P_{2}(L) / P_{2}(F)\right| \sim 0.8$, что также означает более сильное влияние локальных колебаний на температурную

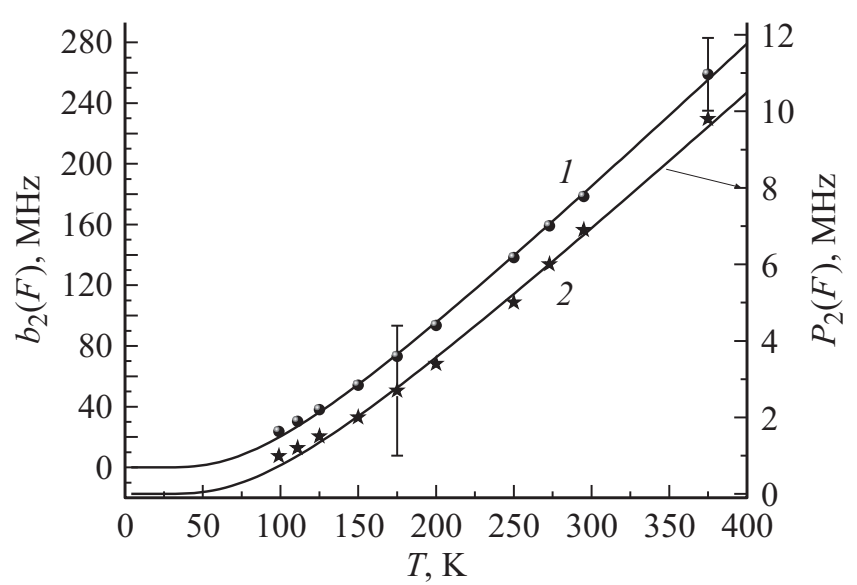

Температурные зависимости $b_{2}(F)(1), P_{2}(F)(2)$. Кривые 1 и 2 описываются выражением (4) с параметрами, приведенными в табл. 2. Положения точек и звездочек определено согласно (1) и (3). Приведенные ошибки включают максимальную экспериментальную ошибку в $b_{2}^{0}(T)$ и $P_{2}^{0}(T)$ плюс разброс в $b_{2}(L)$ и $P_{2}(L)$ из-за неточностей при расчете параметров решетки. 
зависимость КВ. Поскольку эти два механизма (фононно-индуцированный и решеточный) дают вклады разного знака в $Z_{2}^{0}(T)$, они частично компенсируют друг друга, что наиболее заметно в величинах $P_{2}^{0}(T)$.

Таким образом, мы видим, что положительные величины СФВ $b_{2}(F)$ и $P_{2}(F)$ описываются (4) с частотами $\omega$, совпадающими в пределах ошибок. Исходя из этого результата, мы считаем, что однозначное описание температурных зависимостей для вышеуказанных параметров указывает на правомерность использования модели Пфистера [27], предполагающей доминирующую роль локальных колебаний примесного кластера в фононно-индуцированном вкладе. Заметим, что существенное влияние локальных колебаний на процессы диссипации энергии от ПЦ в решетку отмечено также в оптическом диапазоне, что влияет на эффективность работы практических устройств (см. работу [28] и ссылки).

4.3. Температурная зависимость $b_{n}^{m}$. Параметры $b_{4}^{m}(T)(m=0,4)$ анализировались только в „модели жесткой решетки“ [24], поскольку нет хорошей модели для определения $b_{n}^{m}$, а модель Ньюмена [13-15] для их расчета дает неоднозначные результаты, как это было показано ранее в [7]. Взяв (2), (3), (4) из [24] для трех моделей СФВ, мы получили величины $b_{4}^{m}(R L)$, средние значения которых приведенные в табл. 2 .

\section{5. Заключение}

Анализ экспериментальных зависимостей параметров СГ $b_{2}^{0}(T)$ и $P_{2}^{0}(T)$ для примесного центра $\mathrm{Gd}^{+3}$ (нечетный изотоп) в $\mathrm{SrMoO}_{4}$ показал, что учет температурных изменений вклада статической решетки $\Delta Z_{2}(L)<0$ позволил однозначно и с минимальным числом модельных параметров описать температурное поведение спин-фононного взаимодействия $b_{2}(F)$ и $P_{2}(F)>0$. Из трех моделей СФВ наиболее подходящей оказалась модель локальных колебаний примесного кластера Пфистера [27], причем частоты локальных колебаний для $b_{2}(F)$ и $P_{2}(F)$ совпадают (в пределах ошибок). Отношение спин-фононного вклада к температурным изменениям решеточного вклада $\sim 1.7$ в области линейной зависимости для $b_{2}^{0}(T)$ и $\sim 1.25$ для $P_{2}^{0}(T)$. Это указывает на большее влияние на температурное поведение начального расщепления колебаний примесного кластера, чем расширение решетки. Поскольку это вклады разного знака, они частично компенсируют друг друга в широкой области температур, что заметно ослабляет зависимость $Z_{2}^{0}(T)$. Отметим также, что эти результаты аналогичны полученным для $\mathrm{Gd}^{3+}{ }_{\text {в } \mathrm{CaMoO}_{4}}$ и $\mathrm{CaWO}_{4}$.

\section{Список литературы}

[1] L.-Y. Zhou, J.-S. Wei, F.-Z. Gong, J.-L. Huang, L.-H. Yi. J. Solid. State Chem. 181, 1337 (2008).

[2] A. Khanna, P.S. Dutta. J. Solid. State Chem. 198, 93 (2013).
[3] A.S. Shcherbakov, A.O. Arellanes, S.A. Nemov. Opt. Engineering 52, 064001, (2013).

[4] В. Осико, И. Щербаков. Фотоника 39, 14 (2013).

[5] H. Bhang, R.S. Boiko, D.M. Chernyak, J.H. Choi, S. Choi, F.A. Danevich, K.V. Efendiev, C. Enss, A. Fleischmann, A.M. Gangapshev, L. Gastaldo, A.M. Gezhaev, Y.S. Hwang, H. Jiang, W.G. Kang, V.V. Kazalov, N.D. Khanbekov, H.J. Kim, K.B. Kim, S.K. Kim, S.C. Kim, Y.D. Kim. Y.H. Kim, V.V. Kobychev, V.N. Kornoukhov, V.V. Kuzminov, V.M. Mokina, H.S. Lee, J.I. Lee, J.M. Lee, K.B. Lee, M.J. Lee, M.K. Lee, S.J. Lee, J. Li, X. Li, S.S. Myung, A.S. Nikolaiko, S. Olsen, S.I. Panasenko, H. Park, D.V. Poda, R.B. Podviyanuk, O.G. Polischuk, P.A. Polozov, S.S. Ratkevich, Y.Satou, J.H. So, K. Tanida, V.I. Tretyak, S.P. Yakimenko, Q Yue, Y. Yuryev. J. Phys. Conf. Ser. 375, 042023 (2012).

[6] А.Д. Горлов. ФТТ 57, 1371 (2015).

[7] А.Д. Горлов. ФТТ 59, 559 (2017).

[8] W.M. Walsh, jr. Phys. Rev. 114, 1473 (1959).

[9] W.M. Walsh, jr., J. Jeener, N. Bloembergen. Phys. Rev. 139, A1338 (1965).

[10] А. Абрагам, Б. Блини. Электронный парамагнитный резонанс переходных ионов. Мир, М. (1972). Т. 1. 651 с.

[11] Б. Блини. В сб.: Сверхтонкие взаимодействия в твердых телах / Под ред. Е.А. Турова. Мир, М. (1970). С. 15.

[12] С.А. Альтшулер, Б.М. Козырев. Электронный парамагнитный резонанс соединений элементов промежуточных групп. Наука, М. (1972). 672 с.

[13] D.J. Newman, W. Urban. Adv. Phys. 24, 793 (1975).

[14] G.G. Siu, D.J. Newman J. Phys. C 15, 6753 (1982).

[15] D.J. Newman J. Phys. C 8, 1862 (1975).

[16] L.I. Levin, A.D. Gorlov. J. Phys.: Cond. Matter. 4, 1981 (1992).

[17] V.T. Deshpande, S.V. Suryanarayana. Acta Cryst. A 28, 94 (1972).

[18] A. Senyshyn, H. Kraus, V.B. Mikhailik, V. Yakovyna. Phys. Rev. B 70, 214306 (2004).

[19] S.N. Achary, S.J. Patwe, M.D. Mathews, A.K. Tyagi. J. Phys. Chem. Solid. 67, 774 (2006).

[20] A. Senyshyn, H. Kraus, V.B. Mikhailik, L. Vasylechko, M. Knapp. Phys. Rev. B 73, 014104 (2006).

[21] E. Gurmen, E. Daniels, J.S. King. J. Phys. Chem. Phys. 55, 1093 (1971).

[22] W.C. Zheng, S.Y. Wu. Physica B 304, 137 (2001).

[23] R.D. Shennon. Acta. Crystallogr. A 32, 751 (1976).

[24] T. Rewajt, J. Kuriata, J. Typek, J.Y. Buzare. Acta Phys. Pol. A 84, 1143 (1993).

[25] C.Y. Huang. Phys. Rev. 159, 683 (1967).

[26] K.N. Shrivastava. Phys. Rev. 187, 446 (1969).

[27] G. Pfister, W. Draybrodt, W. Assmus. Phys. Status Solidi B 36, 351(1969).

[28] M.P. Hehlen, A. Kuditcher, S.C. Rand, M.A. Tischler. J. Chem. Phys. 107, 4886 (1997). 